\title{
Modelo holístico de planificación curricular para institutos y colegios universitarios de tecnología
}

\author{
Holistic model of curricular planing for institutes and graduate level \\ technology institutions
}

\author{
a Felipe Antonio Bastidas Terán \\ fabastidas@gmail.com \\ Universidad de Carabobo, Venezuela
}

Artículo recibido en septiembre 2018 Arbitrado en octubre 2018

Publicado en enero 2019

\section{Resumen}

El presente trabajo tuvo como objetivo desarrollar un modelo de planificación curricular, basado en los principios de la holística y en los aportes de la hologogía. Se hizo una investigación proyectiva, con un diseño de fuente mixta (documentos y fuentes vivas), en el Instituto Universitario de Tecnología de Caripito, en el estado Monagas, Venezuela. Se aplicaron entrevistas a los profesores, un cuestionario estructurado a los estudiantes, y se revisaron los diseños curriculares de los programas vigentes para el momento. Para determinar los requerimientos del sector laboral de la región, se estudió la oferta laboral de la zona y se hizo un estadio predictivo a fin de anticipar tendencias futuras de demanda laboral. El resultado fue la construcción del Modelo holístico de planificación curricular (Mhoplan), que presenta un proceso continuo y organizado, con énfasis en la investigación, e incorpora seis etapas en un bucle recurrente: Diagnóstico, Viabilidad, (Re) Diseño, Aplicación, Evaluación y Fortalecimiento.

\footnotetext{
Abstract

The present work aimed to develop a curriculum planning model, based on the principles of holistics and the contributions of hologogy. A projective investigation was carried out, with a mixed-source design (documents and live sources), at the Caripito University Institute of Technology, in the state of Monagas, Venezuela. Interviews with the teachers, a structured questionnaire were applied to the students, and the curricular designs of the programs in force at the time were reviewed. To determine the requirements of the region's labor sector, the labor supply of the area was studied and a predictive stage was made in order to anticipate future trends in labor demand. The result was the construction of the Holistic Curriculum Planning Model (Mhoplan), which presents a continuous and organized process, with an emphasis on research, and incorporates six stages in a recurring loop: Diagnosis, Feasibility, (Re) Design, Application, Evaluation and strengthening.
}

\section{Palabras clave:}

Educación; currículum; diseño curricular; hologogía; modelo de planificación curricular
Keywords:

Education; curriculum; curricular design; hologogy; model for curricular planning 
INTRODUCCIÓN

La visión de la sociedad como una sumatoria de individuos y no como la integralidad $\mathrm{o}$ totalidad de relaciones transpersonales hace eterno el debate de si la educación debe atender a la sociedad o a la persona. A través de un abordaje holístico de la educación es preciso observar que sociedad, Estado, cultura y personas son una sola realidad, una totalidad, una integralidad. Los intereses, tendencias y necesidades de las personas, aunque únicos, son para o por el contexto al cual están integrados.

Desde su nacimiento cada persona tiene la necesidad de desenvolverse, proyectarse y trascenderse en la cultura a la que pertenece; sus intereses (diversos o matizados) son comunes en correspondencia con su contexto. Esto no quiere decir que se anulen sus capacidades, tendencias, expectativas y preferencias, pues éstas sólo pueden desarrollarse, potenciarse, proyectarse y complementarse dentro del holos y la complejidad de las relaciones que se dan en su contexto socio - histórico.

En este punto, Barrera Morales (1999) ha desarrollado el concepto de hologogía, a partir del cual la educación se toma en cuenta como un continuo, donde la persona de acuerdo a sus necesidades (que en efecto son sociales) y a sus propias circunstancias particulares (dadas por el contexto), demanda una serie de conocimientos de acuerdo a su experiencia, motivada por sus expectativas, cualidades, propensiones que se complementan con las de sus congéneres. La hologogía se basa en una concepción integral del tiempo y sobretodo de la persona, la cual independientemente de su edad tiene aspiraciones y necesidades de realización pues es un ser en devenir, integrado a su vez dentro de un contexto socio - histórico. La educación es un proceso, un continuo (Barrera Morales, 2001).

En esta trascendencia, el Estado ya no es el dador de la educación, es el facilitador de dicho proceso, como cualquiera otra sinergia de la sociedad. El Estado no puede representar o interpretar los intereses o las necesidades cognoscitivas de las personas o los grupos, porque éstos son quienes lo constituyen. Las personas y los grupos como integrantes del Estado son quienes determinan la política (concordancia, consenso e integralidad de medios y fines), o el proceso, o la sistematicidad de los conocimientos requeridos por ellos para perfeccionarse o bien desarrollarse.

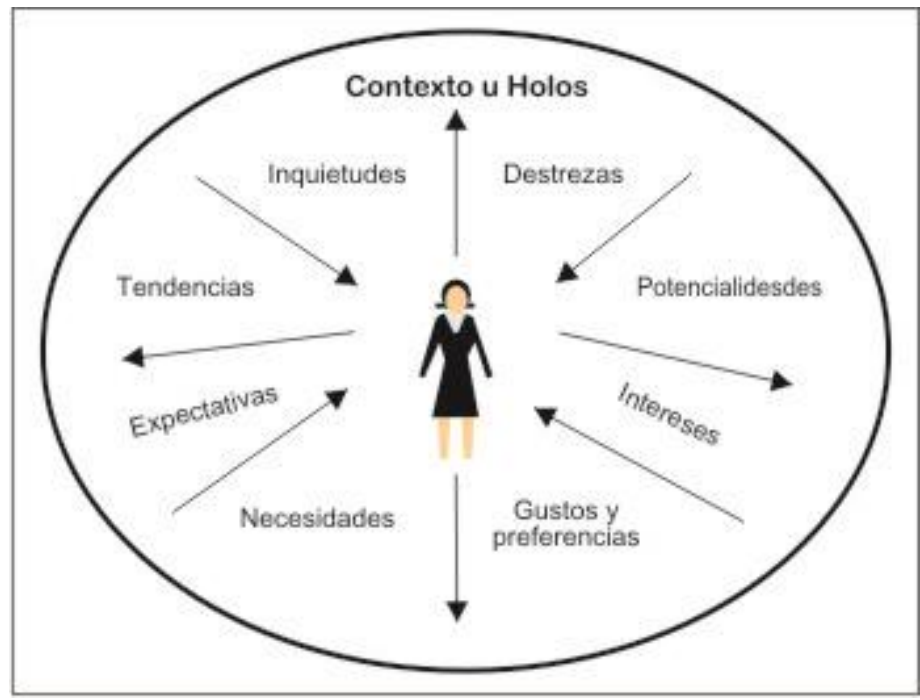

Figura 1. La persona como integralidad y complemento de su contexto 
La hologogía está más acorde con los retos que plantea la sociedad del siglo XXI. En este sentido, la finalidad de la educación es permitir seguir conociendo, pero partiendo de lo ya conocido (Barrera Morales, 2001). Por eso la investigación es clave dentro de esta concepción, pues más que impartir conocimientos, se trata de compartir, innovar y generar instrumentos para crear nuevas fuentes del mismo. Es preciso introducir los cambios pertinentes en la concepción de la educación en las instituciones de educación formal, como punto de partida para el mejoramiento y perfeccionamiento de la sociedad. Por ello, el propósito de este trabajo fue proponer un modelo de planificación curricular basado en la hologogía.

Objetivo que requirió de una valoración de la problemática, pues la educación del siglo $\mathrm{XX}$ quedó supeditada al desarrollismo económico, es decir, la pertinencia consistía en satisfacer los requerimientos profesionales del empresariado, las universidades casi se convirtieron en una empresa burocrática que "producía recursos humanos". Esta concepción está seriamente cuestionada gracias a los estudios pronósticos del futuro.

Uno de los cambios más importantes es la concepción del empleo. Las nuevas tecnologías han propiciado otras modalidades de contratación de personal. Esto se sustenta sobre la base del crecimiento del empleo por contrato a tiempo parcial. Así se tiene: a) teletrabajo, b) trabajo en el hogar, c) trabajadores móviles, d) surgimiento de corporaciones virtuales, y e) telemercadeo.

Debido a este proceso dinámico y al cambio vertiginoso de las tecnologías, la empresa ahora debe mantener una capacitación constante, gran parte de su personal ya no está en un sitio específico. Tiene como alternativa propiciar condiciones favorables para un autodesarrollo profesional. Esto permite un amplio margen de flexibilidad y genera mayor autoresponsabilidad de los profesionales.

El trabajo incondicional de por vida o el desarrollo en una sola empresa tiende a desaparecer. Lo anterior implica una reducción de las gerencias medias lo que genera un techo para los graduados universitarios. Las empresas se están quedando con mano de obra semicalificada y pequeñas elites gerenciales.

Esto conlleva a la búsqueda de actividades profesionales autogestionarias fuera de la empresa. En primer lugar, los profesionales universitarios pueden crear pequeñas empresas de servicios extras y temporales para grandes empresas e incluso para servicios públicos del Estado. Otra alternativa pudiera ser la prestación de servicios culturales y sociales no cubiertos o cubiertos parcialmente por el Estado, como el cuidado de niños y ancianos, jóvenes con problemas de conducta, servicios ambientales, servicios culturales, servicios educativos informales, recreación y entretenimiento, apoyo al pequeño comercio (Gamus, 1998).

Estas iniciativas pueden estar financiadas mediante convenios entre Estado/ empresas/comunidad/personas. Lo anterior ilustra como los límites entre organizaciones, familias y personas se diluyen propiciando un mayor desarrollo personal y comunal; es decir, se está trascendiendo la visión fragmentaria de sociedad-Estado. Se está percibiendo como una integralidad y complejidad de relaciones transpersonales. Una vez más es necesaria la percepción holística de la complejidad de las relaciones entre personas y su contexto, la globalización exige la integración de comunidad, sociedad, Estado, cultura y mundo. 
A la luz de este razonamiento, la educación está más centrada en la preparación de la persona que le permita desarrollarse en múltiples áreas laborales y sociales, con una base para el autoaprendizaje. Además existe una tendencia en el ámbito no sólo laboral sino en cualquier contexto en los cuales las líneas gerenciales, técnicas y administrativas se desdibujan, se trascienden, se complementan, tal como lo propone la holística.

En este sentido, no se trata de acumular conocimiento, sino más bien, cómo seleccionarlo, organizarlo y sistematizarlo de acuerdo a intereses propios, lo que conlleva a un cambio en los modelos de aprendizaje. La calidad de los conocimientos adquiridos se valorarán más en función de:

- Capacidad de un aprendizaje permanente

- Desarrollar actitudes tales como:

a) autonomía,

b) autoresponsabilidad,

c) trabajo en equipo y cooperación,

d) apertura y adaptabilidad al cambio,

e) creatividad e innovación,

f) capacidad de identificar y resolver problemas,

g) capacidad de comunicación, y

h) autoestima.

No obstante, estas son tan sólo tendencias, la única certeza que se tiene con respecto a la nueva sociedad del conocimiento es su carácter diverso y complejo, con un sin fin de nuevas probabilidades de desarrollo. En este caso el profesional requerido es una persona capaz de adelantarse a esos cambios y de observar oportunidades en lo que parecen situaciones complejas o problemáticas. Se requiere de profesionales transdisciplinarios y con una percepción holística de la realidad.
Por otra parte, los retos de conocimiento se hacen necesarios en América Latina en contextos que requieren de su óptima utilización: organización de la sociedad civil, nuevas formas de producción, preservación del ambiente y de la cultura, nuevas tecnologías alimentarias, desarrollo autosostenido, generación de nuevas fuentes de empleo. Por consiguiente, la pertinencia estará basada sobre los conocimientos universales (ciencia y tecnología) y contextualizados en relación con toda la vida local con necesidades sociales y económicas (García, 1998).

La holística es imprescindible en la forma de abordar el problema educativo, ya que toma en cuenta a la persona como integrante de un contexto amplio, tal como lo van indicando los cambios generados en el proceso de globalización. Sin parcelamientos ni fragmentaciones sino como una realidad compleja y al mismo tiempo integral.

La percepción fragmentaria y dual de la sociedad ha generado no solamente las contradicciones conceptuales con respecto a la educación, sino también del procedimiento, de los medios, de los objetivos y de los fines de la misma, es decir, con respecto a la política educativa y al currículo. Para Angulo y Blanco (200o) las diferentes acepciones de currículo responden a modelos epistémicos de diversa índole.

De igual manera, las formas dicotómicas de abordar la realidad, como teoría - práctica, planificación acción, objeto - sujeto, causa - efecto, han sido trasladadas a la definición de currículo. De esta forma, el currículo posee una "parte concreta", otra "práctica" y otra de "investigación".

El currículo es una trascendencia de todas estas fragmentaciones que han sido vistas por algunos autores desde una aproximación integral, mas no trascienden 
tal como es menester en holística. Para Meza (1980), "el aprendizaje debe desarrollarse como un proceso de interacción vital con el marco totalizador del mundo que rodea al alumno" (p. 15).

La persona no es un ser aislado, sino que está integrado o pertenece a un contexto, por lo tanto sus preferencias, inquietudes e intereses se complementan y potencian con y en su entorno: "Una visualización integral del currículo y el manejo necesario de una metodología para su estudio, permiten pensar que a través de él es posible abordar el sentido del fenómeno educativo en su totalidad" (Meza, 1980, p. 26).

En efecto, al realizar un abordaje holístico del currículo se produce otro holístico de la educación, o viceversa. La totalidad que es la sociedad, supone de igual manera una integración de lo que es su proceso educativo. No hay necesidad de fragmentar o dividir lo que es currículo y quienes lo integran. De esta manera, "el trabajo del administrador educacional (docente) consiste en planificar, diseñar e implantar un sistema eficiente y eficaz que responda a las necesidades de los alumnos y la sociedad" (Kaufman, 2000: 23).

Como se dijo anteriormente, las necesidades personales y sociales son un complemento, una integralidad. Siguiendo con Kaufman (2000) "las variables de acción recíproca del hogar, el vecindario, la cultura y la sociedad, deben incluirse en el diseño de la educación, puesto que, cualesquiera que sean nuestros motivos, se nos sigue considerando responsables" (p.24). Las variables que menciona este autor no son más que sinergias de un mismo evento, pues todas integran una misma realidad. Todos son responsables del currículo, pues él atiende a necesidades y potencialidades complementarias de las personas.

Las necesidades de conocimiento de la persona se complementan dentro de holos mayores. Para satisfacerlas es preciso que todas las sinergias se involucren y participen, en el caso de la educación, el currículo debe ser diseñado de forma integral $y$ trascendente para lograr el efecto deseado: satisfacer las necesidades de conocimiento personales, que en realidad son las mismas del hogar, la comunidad, la escuela, la sociedad o la cultura o el Estado, las cuales son sinergias de un holos mayor.

El currículo es el esfuerzo sistemático, progresivo y trascendente del hogar, la comunidad y la escuela (estudiantes, profesores y administradores) para compartir y producir conocimientos que satisfagan las necesidades, capacidades, expectativas, tendencias y potencialidades de las personas, las cuales se vendrían a complementar y proyectar dentro de holos mayores, que generalmente se denominan sociedad, cultura o Estado. En este caso el currículo es un proceso "original, particular, único y distinto, frente a una situación educativa dada" (Meza, 1980).

El currículo contempla las metas, procedimientos y fines compartidos por una comunidad (integrante de una sociedad). Es decir, es una política, una dinámica de transmisión, comunicación y producción de conocimientos, que generalmente tiene su asiento en la escuela o la academia, como lugar específico en el cual se ejecuta la mayoría de estas actividades. De acuerdo a lo anterior, currículo es en sí planificación.

Kaufman (2000) sostiene que "un enfoque sistemático educativo orientado a la acción, requiere que se realice una planificación formal y sistemática, lo mismo que diseños, aplicaciones, evaluaciones y revisiones" (p.14). Hablar de planificación curricular es entonces hablar de currículo. Sin embargo, muchas veces el currículo no posee un diagnóstico y carece de algún diseño o plan, y menos contempla una evaluación. Esto sucede con mucha 
regularidad en las instituciones educativas de Venezuela. Para mejorar esta situación hay que tomar en cuenta que la planificación curricular debe partir de la investigación (Meza, 1980). De esta forma el currículo para ser pertinente en la actualidad debe estar sustentado en la ciencia.

La planificación curricular no sólo se manifiesta en su sustento investigativo, también se expresa a través de programas pues diseña líneas atendiendo a un plan mayor, pero también, a un contexto específico, o puede decirse currículo es una meso política que adapta los lineamientos de una macro política atendiendo a los requerimientos de una comunidad específica (Beauchamp, 1981 como se citó en Angulo y Blanco, 2000) (ver figura 2).

Así mismo, el currículo es agente de comunicación entre las sinergias que intervienen en él, personas, docentes, hogar, comunidad, escuela, sociedad (Estado o cultura).

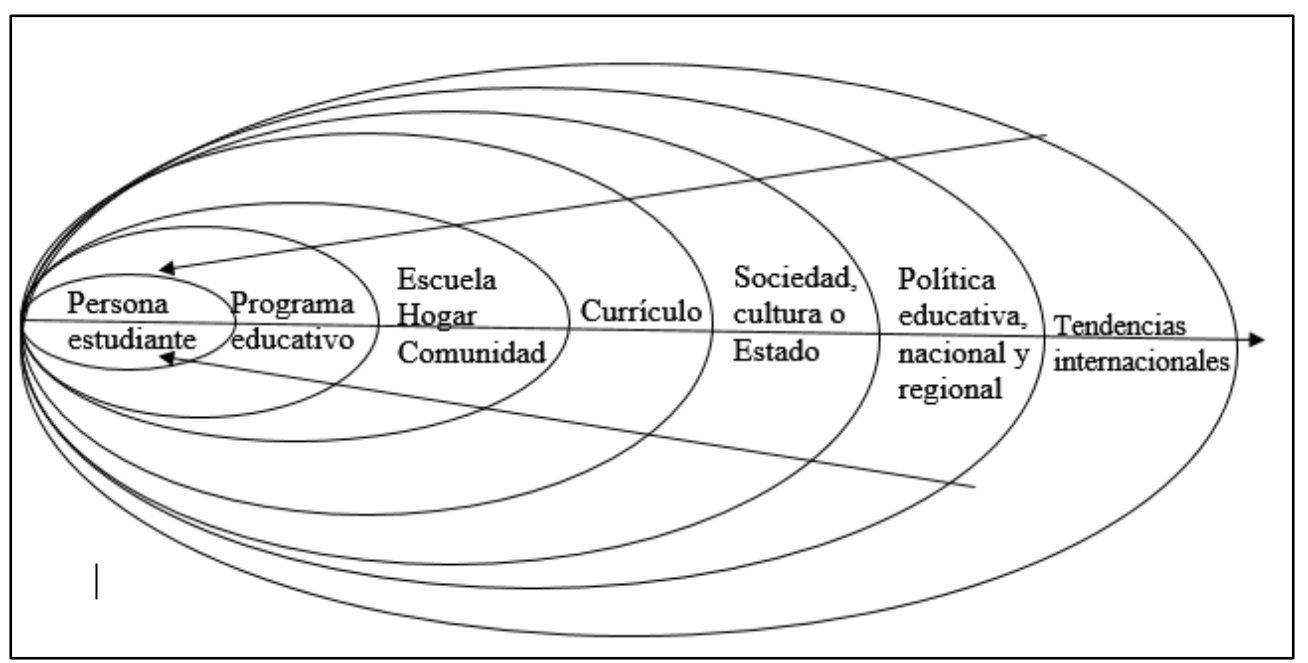

Figura 2. El currículo como política

El currículo es demandante y al mismo tiempo productor de política. Es él quien advierte los cambios y transformaciones requeridas por las comunidades y las personas (dimensión evaluativa de la política), las cuales reciben presiones de su contexto (local, regional, nacional y global); y por otro lado, es responsable de instruir a las personas y las comunidades para que ejecuten y dinamicen esos cambios $y$ transformaciones "el currículo es una conclusión deducida de adaptación al cambio social" (Díaz -Barriga, 1998). También sirve como detector de nuevas posibilidades y potencialidades de desarrollo.
Ante los requerimientos y posibilidades de transformación y cambio en América Latina, dada por la acumulación progresiva de problemas sociales irresolutos (desde la Colonia hasta hoy, generados por patrones y modelos descontextualizados), y dados por el proceso de globalización y la sociedad del conocimiento, es el currículo el instrumento fundamental para propiciar esos cambios y desplegar posibilidades y potencialidades.

Todo currículo debe estar orientado a lograr el perfil que se requiere de los profesionales en formación. En este sentido, de acuerdo a las exigencias del mundo globalizado, interdependiente, cambiante y complejo de hoy, la pertinencia educativa de 
los tecnológicos debe estar sustentada sobre una formación integral del egresado. El perfil del tecnólogo egresado debe tener las siguientes características: investigador, creador, innovador y gerente de tecnologías.

A tal efecto, se han de tomar en cuenta las dimensiones que de forma integral y trascendente manifiesta el ser humano, a saber:

La dimensión intelectiva: "Comprende la capacidad de razonar, el pensamiento, la abstracción, la creatividad, la intuición, la inferencia y la conceptualización" (Hurtado, 2001). Los tecnológicos deben promover, fomentar y potenciar las competencias de creación e innovación tecnológica de las personas; es decir, abrir múltiples posibilidades de creación e innovación de modelos, sistemas, programas, organizaciones, procesos, diseños, prototipos $\mathrm{y}$ tecnofactos que de manera armónica $\mathrm{y}$ pertinente re ordenen el contexto venezolano y latinoamericano de acuerdo a nuestros valores, requerimientos $y$ potencialidades.

Para lograr tal cometido es imprescindible desarrollar competencias investigativas que puedan generar y soportar la creación de tecnologías novedosas.

La dimensión biofisiológica "Comprende todo lo relacionado con lo corporal... todos los aspectos de orden biofisiológico como el sueño, la alimentación, la motricidad..." (Hurtado, 2001). Esto se relaciona en gran medida con la formación integral del tecnólogo. En primer lugar, no se debe descuidar la salud y el equilibrio emocional que deben poseer las personas para vivir en un mundo complejo, cambiante y pleno de oportunidades en situaciones que quizás parezcan contradictorias o paradójicas. Los tecnológicos deben fortalecer estas condiciones personales mediante actividades deportivas y artísticas y asistencia médica y psico social. En segundo lugar, no basta con crear tecnologías: es necesario que los tecnólogos tengan las competencias necesarias para determinar la infraestructura, personal, recursos y financiamiento que permitan la difusión o reproducción de tecnologías, así como su innovación y perfeccionamiento.

La dimensión volitivo-social se vincula con

la voluntad, la motivación, la toma de decisiones, la intencionalidad y las actitudes, la responsabilidad, las aspiraciones y las metas, y en general todos los aspectos de orden psico social que orientan a las personas y su relación con el contexto (Hurtado, 2001, s.n).

Para difundir, promocionar, reproducir o comercializar una tecnología es necesario que sus creadores sean capaces de diseñar la organización que motorice estas actividades. Para este fin un tecnólogo-gerente ha de contar con herramientas claves tales como toma de decisiones, trabajo en equipo, capacidad negociadora, liderazgo, responsabilidad, proyecto de vida definido como tecnólogo autogestionario, entre otros.

Además, el tecnólogo - gerente ha de tener una visión amplia e integradora de su contexto para que pueda desarrollar su modelo, sistema, diseño, programa, proceso, prototipo o tecnofacto de manera armónica y pertinente en su comunidad. También ha de ser capaz de liderar la integración comunidad - gobierno - empresa como vía de solución para mejorar los procesos sociales actuales.

La dimensión ética y moral: "tiene que ver con el sentido de la vida, los valores..." (Hurtado, 2001). La realización del tecnólogo latinoamericano tiene que estar en estrecha relación con valores fundamentales $y$ transformadores de nuestro devenir socio histórico, caracterizado principalmente por las enormes desigualdades sociales. Tales 
valores cardinales son: responsabilidad, para crear tecnologías de bajo impacto ecológico, sensibilidad y comprensión de procesos sociales, cooperación, y sobre todo respeto y tolerancia.

En sintonía con estas ideas, el modelo propuesto parte y se soporta en el pensamiento holístico, como herramienta idónea para responder a las competencias integrales demandadas a los profesionales tecnológicos.

Sobre la base de estas dimensiones, la Holística también tiene aplicaciones para iniciar y desarrollar en las personas el pensamiento complejo e integral necesario para afrontar los retos del siglo XXI. Según Fernández (2001), la Espiral Holística, diseñada por Hurtado $(1998,2000)$ es una herramienta fundamental para desarrollar el pensamiento complejo en las personas desde que incursionan en la educación formal, es decir, desde la educación pre escolar. Lo mismo vale para la educación tecnológica universitaria según se ha demostrado esta exposición.

\section{MÉTODO}

Para el desarrollo de esta propuesta se tomó como referencia el Instituto
Universitario de Tecnología de Caripito, en el estado Monagas, Venezuela. Se hizo una investigación proyectiva, con un diseño de fuente mixta (documentos y fuentes vivas).

Participaron como informantes profesores de los diferentes programas, con quienes se hicieron varios encuentros grupales para obtener información. También se aplicó un cuestionario estructurado a los estudiantes y se revisaron los diseños curriculares de los programas. Para determinar los requerimientos del sector laboral de la región, se estudió la oferta laboral de la región y se hizo un estadio predictivo a fin de anticipar tendencias futuras en el campo profesional de los egresados. A partir del análisis de la información recolectada, se procedió a diseñar el modelo.

\section{RESULTADOS}

De acuerdo a lo anteriormente expuesto se propuso un modelo holístico de planificación curricular (Mhoplan) que explique y oriente las acciones investigativas, de tal manera que aseguren la pertinencia y calidad educativa en un contexto cambiante y complejo.

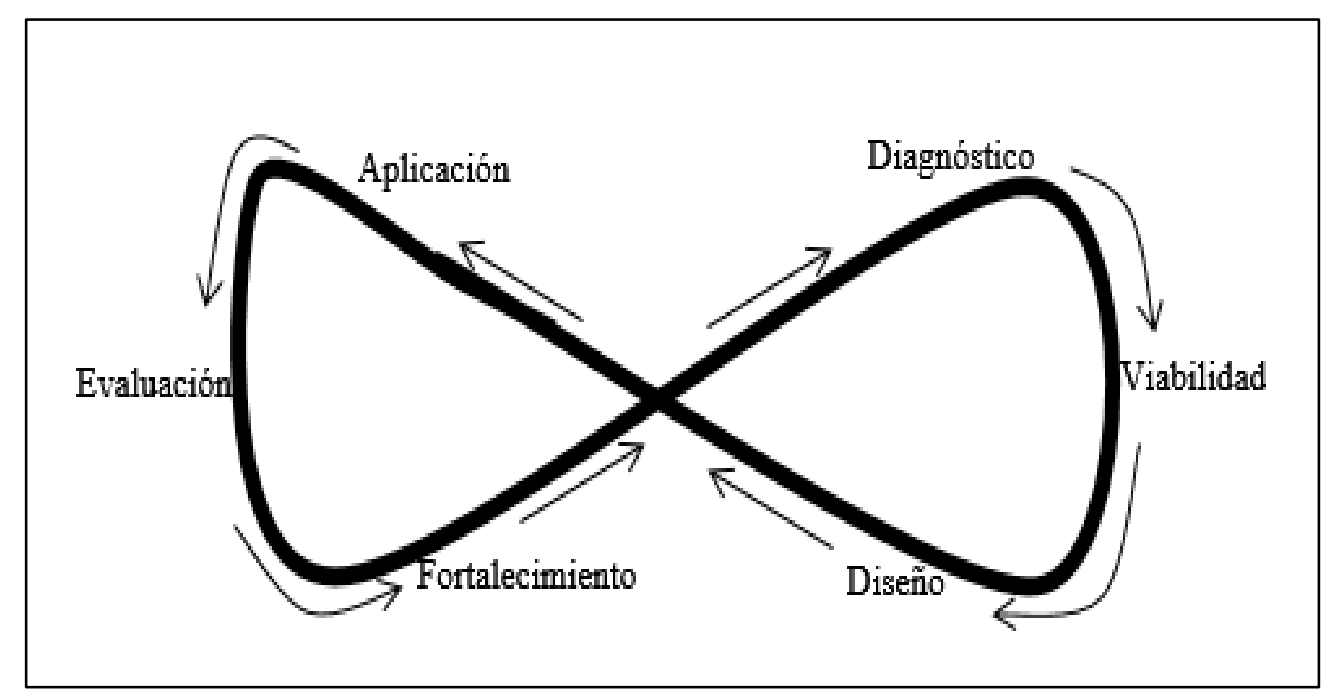

Figura 3. Modelo Holístico de Planificación Curricular 
El Mhoplan es un proceso continuo, dinámico, multidimensional, trascendente e integrativo que se basa en las siguientes fases o momentos estelares: Diagnóstico, Viabilidad, (Re) Diseño, Aplicación, Evaluación y Fortalecimiento. Para asegurar la pertinencia educativa se ha de cumplir permanentemente el paseo por todas las fases. Al llegar al momento de fortalecimiento curricular se inicia un nuevo ciclo infinito que comienza en el diagnóstico, luego viabilidad. Cada infinito completo integra y trasciende los conocimientos adquiridos en infinito anterior (figura 3).

\section{Fase I: Diagnóstico}

En esta fase la comunidad tecnológica se abre y se despliega en su contexto con la finalidad de observar y detectar las necesidades, posibilidades y potencialidades de educación tecnológica para definir los perfiles profesionales pertinentes. Los tecnológicos generalmente abarcan esta fase en su fundación, pero difícilmente lo cubren nuevamente. Se comienza realizando visitas preliminares a empresas y comunidades, se revisa si hay cambios socioeconómicos, sociopolíticos y si hay nuevos cambios en los planes de desarrollo que demanden el concurso de nuevos profesionales.

Las necesidades $\mathrm{o}$ potencialidades educativas se detectan, en las empresas, a través de indicios muy sutiles como cargos sin ocupar o cubiertos con otros perfiles, si hay rediseños organizacionales o si las empresas se han expandido o si se han creado nuevas. También se observa el contexto, se aborda en su complejidad para aprehender potencialidades no percibidas anteriormente.

Luego, se establecen las relaciones y sinergias de los diversos requerimientos y potencialidades educativas, para integrarlos en perfiles preliminares, los cuales serán revisados y evaluados en compañía del empresariado, el gobierno y la comunidad.

Una vez establecidos y delineados los perfiles preliminares por la institución se procede a compararlos con los de otras instituciones regionales. Si las coincidencias son pocas se procede a tomar aspectos de los perfiles ajenos como orientación, pero siempre observando que no exista duplicidad de oferta. Si las coincidencias son considerables, se delinean nuevas modalidades para ofertar la carrera, como educación a distancia, semipresencial, postgrado o cursos de actualización; la idea es complementar mas no solapar oferta.

Si las coincidencias son muchas, o el perfil es muy similar al que oferta otra institución regional, se prorroga la consideración de su apertura; en este último caso se puede seguir con el proceso, si y sólo si la demanda de educación media es alta y no puede ser cubierta por una sola institución.

\section{Fase II: Viabilidad}

Se comienza a recoger indicadores de la viabilidad de los perfiles preliminares mediante dos procesos:

En primer lugar, los criterios de estudios de viabilidad tienen que ver con las potencialidades de la institución. Se mide la correspondencia entre los requerimientos de infraestructura, de equipos, de personal, organizacionales y financieros de los nuevos perfiles y los recursos y potencialidades de la institución.

$\mathrm{Si}$ existe correspondencia entre los recursos del nuevo perfil y los institucionales, quiere decir que el perfil o los perfiles son viables. $\mathrm{Si}$ no hay correspondencia el o los perfiles no son viables a corto plazo, en este caso, se realiza un proyecto de financiamiento, para ir acondicionando la institución según el 
requerimiento del perfil preliminar.

En segundo lugar, se pronostica el cambio de los requerimientos del perfil en el tiempo y la estimación de la demanda de educación media del perfil en el futuro.

$\mathrm{Si}$ los requerimientos de los profesionales aumentan en el tiempo la carrera es viable. Si se detectan cambios o vulnerabilidad del requerimiento profesional ante los cambios sociales y económicos, se debe prever de antemano la secuencia y la profundidad de la revisión y rediseños de las carreras. Si el requerimiento a largo plazo tiende a descender, la carrera es poco viable; en este caso se pueden abrir cohortes puntuales en la modalidad de postgrado o cursos de actualización, en las cuales se puede trabajar con poca matrícula.

En cuanto a la demanda futura de educación media de carreras afines, si ésta va en aumento o es estable la carrera es viable. $\mathrm{Si}$ por el contrario va en descenso es poco viable.

Se comparan y se ponderan ambos criterios. Si la demanda es alta o estable y los requerimientos profesionales de las carreras son estables o van en aumento la carrera es altamente viable; aún más si coinciden con los objetivos, misión y visión de la institución (sobre todo en lo concerniente a la aprobación legal). En caso contrario, se toman las previsiones y se proyectan los recursos necesarios que aseguren la permanencia de la carrera, ya sea por el aumento de la matrícula o a los cambios en el requerimiento profesional (rediseños).

Muchas instituciones no toman en cuenta estos aspectos, y en determinado momento, no pueden responder a las exigencias de las carreras, sobre todo aquellas que necesitan de infraestructura amplia como laboratorios y equipos de alta tecnología. Este caso se ve en Venezuela en las carreras que tienen que ver con producción audio visual (Comunicación
Social, Medios Audiovisuales, Arte, Diseño Gráfico, Publicidad), cuyos equipos y materiales de producción además de costosos varían y se innovan rápidamente, y la capacidad de sus instalaciones suele ser rebasada con cualquier aumento leve de la matrícula.

\section{Fase III: Diseño}

Una vez determinada la viabilidad de los perfiles y las acciones a seguir es preciso revisar y construir el basamento teórico, conceptual, epistémico y legal de los mismos; que orientarán sobre métodos y actividades.

Se revisan los cambios de valores y fines de la comunidad, se organiza y se explica el perfil de la carrera de acuerdo a estos y a las potencialidades y condiciones del contexto socio geográfico y socio histórico de la institución. De igual forma se justifica la carrera con la información recopilada hasta la fase anterior y con soportes teóricos e investigativos relacionado con las carreras.

Se analiza y se justifica la carrera de acuerdo a la política de desarrollo regional, a la política educativa y al marco jurídico educacional, así como al marco de leyes referido al área de conocimiento de cada carrera. De igual manera se explican las carreras de acuerdo a la política, perfil, objetivo, misión y visión de la institución; así como de los reglamentos internos.

Luego, se elabora un informe preliminar sobre el basamento epistémico, teórico y legal de las carreras.

Cumplido lo anterior, precisados $\mathrm{y}$ evaluados los objetivos de las carreras, se realiza un listado de competencias profesionales de cada una de ellas y también se especifican las áreas de conocimiento que deben cubrir. En compañía de expertos se establece la modalidad, los períodos, los objetivos de las unidades curriculares (cruce 
de los ejes transversales con los longitudinales) y la duración de la carrera de acuerdo al perfil y a las competencias previamente reseñadas.

Una vez establecidos los objetivos de las carreras, las competencias profesionales, el basamento epistémico, teórico y legal, los objetivos de las unidades curriculares y la duración de la carrera, se procede a realizar el diseño definitivo. Este diseño debe contener: Requisitos del personal docente (especialidad, experticia y habilidades), métodos, técnicas, tácticas y actividades de aprendizaje especificadas por unidades curriculares, recursos (materiales, mobiliario, insumos para labores administrativas y docentes, etc.), de acuerdo a la legalidad $y$ a las competencias profesionales se establecen las prelaciones y las unidades curriculares (de acuerdo a las horas prácticas y teóricas), personal (además del docente, encargados de laboratorios, mantenimiento, asistencia docente, de administración), finalmente la planta física y equipos (laboratorios, talleres, recursos docentes como computadoras, retro proyectores, pizarras, etc.). Se diseñan los programas definitivos de las unidades curriculares.

\section{Fase IV: Aplicación}

Aprobada la carrera, se aplica el diseño curricular y se registran informaciones de su ejecución. Se desarrolla el diseño curricular y se apuntan y registran los resultados derivados de las aplicaciones curriculares en la institución y su entorno (talleres, seminarios, etc.). Al finalizar el primer período se revisa la efectividad de los métodos, tácticas y técnicas (metotactécnicas) de aprendizaje. Se aplica una prueba de conocimiento para medir el logro de los objetivos. Se explican los resultados de acuerdo al basamento legal, teórico y epistémico del currículo. Se toman las previsiones y se diseñan las correcciones a seguir para el cumplimiento de los objetivos generales y específicos de las carreras.

Se continúan los períodos del diseño. Se registra y sistematiza la información de cada una de las unidades curriculares. Se confirma la pertinencia de las correcciones de acuerdo a los objetivos de las unidades curriculares y los objetivos de las carreras. Se siguen aplicando las pruebas de conocimiento al final de cada período, asegurando la adquisición de las competencias profesionales. Se pueden modificar las metotactécnicas e incluso los objetivos y contenidos de algunas unidades curriculares. Se evalúan los resultados y se aplican de nuevo las correcciones.

A medida que avanza la carrera es preciso revisar si va a la par de los cambios en el requerimiento profesional. Se detectan y se describen los cambios del requerimiento profesional mientras se ejecuta el diseño. Se analizan las actividades del desarrollo del diseño curricular en correspondencia de los cambios del requerimiento profesional. Se revisa la precisión de los cambios pronosticados del requerimiento profesional realizados en la fase de viabilidad.

\section{Fase V: Evaluación}

Se registra y sistematiza la información sobre las competencias adquiridas por los estudiantes, mediante tres procedimientos principales: prueba general de conocimiento, presentación de informe de pasantías y trabajos especiales de grado y seguimiento al egresado (por medio del cual se corrobora la ubicación y desempeño profesional de acuerdo al perfil de la carrera). Se analizan, se comparan y se explican los datos recogidos en la fase anterior mediante los objetivos y competencias de la carrera y su basamento legal y teórico - filosófico. Se evalúan los métodos, los recursos y el 
personal (docente y administrativo), así como las instalaciones y los equipos. Culminada la carrera se evalúa el alcance de los objetivos.

\section{Fase VI: Fortalecimiento}

Se diseñan y se introducen los correctivos necesarios de acuerdo a los resultados de la evaluación para que el diseño curricular se ajuste a los cambios del requerimiento. Se replantean objetivos de las unidades curriculares, se crean, se sustituyen o innovan las unidades curriculares y se hace lo propio con los métodos, técnicas y estrategias.

Mientras se ejecuta esta fase ya se vuelve a iniciar el ciclo de las fases diagnóstico, viabilidad y rediseño de la carrera e inmediatamente a un nuevo proceso de aplicación y se vuelve a repetir el ciclo infinito. De esta manera la pertinencia de la carrera se asegura. La propuesta se fundamenta en que, debido a los cambios constantes del contexto y a su complejidad, los tecnológicos deben estar permanentemente rediseñando y revisando sus carreras, es la única manera de responder efectivamente a los requerimientos profesionales con calidad educativa.

La implantación del Mhoplan, requiere para su perfecto desarrollo personal exclusivo, comprendido por el siguiente equipo: Un gerente de investigación, tres investigadores, un relacionista público, un analista de soporte de datos y dos secretarias. Según los basamentos legales este equipo está integrado a la subdirección académica de los tecnológicos. Se sugiere organizarlo como una Unidad de Planificación e Investigación Curricular, adscrita a dicha dependencia.

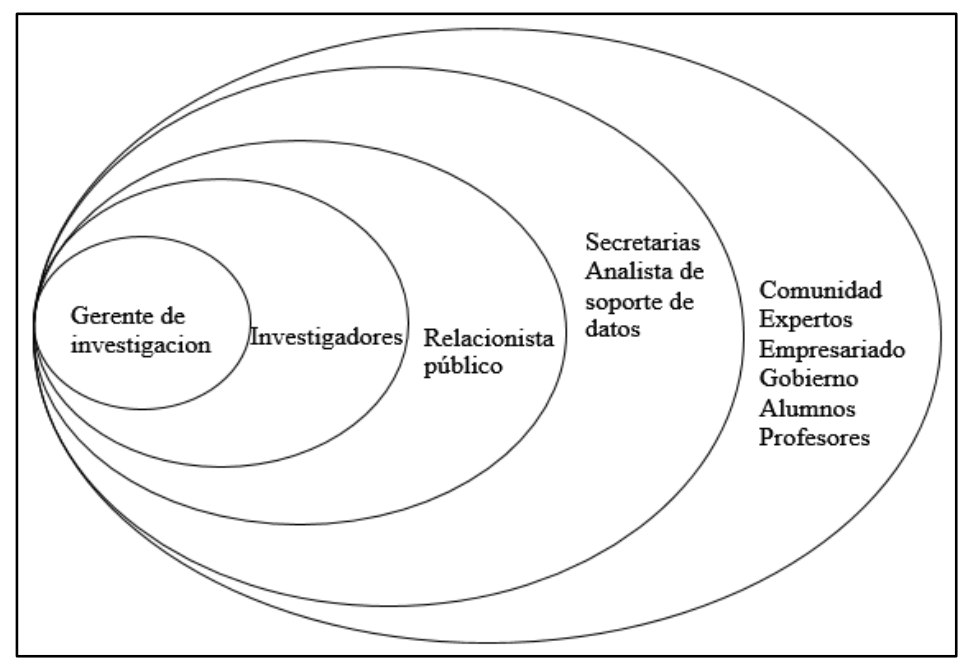

Figura 4. Planificación curricular

Se logró configurar el modelo de planificación curricular a partir de los principios de la holística. Algunos principios de la Holística aplicados en el Mhoplan.

Con respecto al principio de devenir, cada paseo por el modelo, es decir por el ciclo infinito, produce una serie de conocimientos e informaciones que aseguran la pertinencia educativa continua, de esta manera al seguir con el ciclo infinito, se integran $y$ se trascienden dichos conocimientos e informaciones, para establecer un nuevo orden contextual que dé cuenta de las relaciones (cambios) que, al 
finalizar de nuevo el ciclo, exigen un nueva interpretación u ordenamiento curricular.

En cuanto al Principio de continuidad, el Mhoplan es continuo, se pasea constantemente por sus seis fases, pero no es una secuencialidad lineal o un ciclo estacionario. En su recorrido se despliega y abre nuevas posibilidades, es multidimensional.

La revisión curricular, que era vista como una "fase o un requisito obligado" en la planificación curricular, es continua y queda así integrada completamente en el Mhoplan en todos sus momentos estelares.

En referencia al principio de simultaneidad, las fases son momentos estelares, es decir, son procesos que se pueden dar de manera simultánea, por ejemplo, cuando en el diagnóstico se hace el despliegue en el contexto para detectar requerimientos y potencialidades, y se revisan los perfiles preliminares con la comunidad, el gobierno y el empresariado, se está precisando y recabando la información para explicar la carrera de acuerdo a los valores y las potencialidades del contexto socio geográfico $y$ socio histórico, proceso que se cubre por completo en la fase de (re) diseño.

\section{CONCLUSIÓN}

Después de los procesos de investigación realizados, se logró construir el Modelo holístico de planificación curricular (Mhoplan) que, aunque se basó en las indagaciones realizadas en un instituto tecnológico particular, constituye un aporte para reorientar la planificación curricular en otras instituciones. El Mhoplan presenta un proceso continuo y organizado, con énfasis en la investigación, que incorpora seis etapas en un bucle recurrente: Diagnóstico, Viabilidad, (Re) Diseño, Aplicación, Evaluación y Fortalecimiento. Esto implica un seguimiento permanente y una actualización constante del currículo, tal como se requiere en la dinámica del conocimiento del siglo veintiuno.

\section{REFERENCIAS}

Angulo, J. y N. Blanco. (200o). Teoría y desarrollo del currículum. Málaga, España: Ediciones Aljibe

Bastidas, F. (2003). Política institucional de planificación curricular para un instituto universitario de tecnología. Caracas: Sypal

Barrera Morales, M. (1999). Holística, comunicación y cosmovisión. Caracas: Fundación Sypal - Fundacite Anzoátegui

Barrera Morales, M. (2001). Hologogía: Introducción a la educación holística. Caracas:Fundación Sypal

Díaz-Barriga, F. (1998). Metodología de diseño curricular para la educación superior. México: Editorial Trillas

Fernández, I. (2001). "Educación Holística en Educación Media”. II Jornadas de Investigación Holística. Caracas:Universidad Católica Andrés Bello

Gamus, E. (1998). Los desafíos en la educación-trabajo hacia el siglo XXI. Cuadernos del Cendes. Año XV, $\mathrm{N}^{\circ} 37$, Enero - Abril, Caracas

García, C. (1998). La educación superior en Venezuela una perspectiva comparada con el contexto de la transición hacia la sociedad del conocimiento. Cuadernos del Cendes. Año XV, $N^{\circ}$ 37, Enero-Abril, Caracas

García, C. (200o). Investigación y toma de decisiones en educación superior en Nueva Sociedad. $N^{\circ} 165$, Enero - Febrero, Caracas

Hurtado, J. (2000). Metodología de la investigación holística. Caracas: .Fundación Sypal-IUTC

Hurtado, J. (2001). Procesos grupales y psicología de la integración. Sypal: Caracas

INPAHU, Fundación Escuela Superior Profesional (1997). Acervo educativo. Ser y sentido de la formación técnica $y$ tecnológica en las instituciones de educación superior. Bogotá: INPAHU 
Israel, R. (1998). Educación, ciencia y tecnología. Reflexiones de fin de milenio. Santiago de Chile:LOM Ediciones

Kaufman, R. (2000). Planificación de sistemas educativos. México: Editorial Trillas

Meza, C. (1980). Algunas consideraciones sobre currículum escolar. Revista Temas de Educación (Circulación restringida), Caracas: UCV, Facultad de Humanidades y Educación

Quijada G., C. Villarroel y L. Bravo (1985). Material de apoyo para el curso: Diseños curriculares en educación superior. Mimeo, Caracas: UCV, Facultad de Humanidades y Educación

República Bolivariana de Venezuela (1999). Nueva Constitución. Gaceta Oficial $\mathrm{N}^{\circ}$ 36.86o. Año CXXVII - Mes III. Caracas
República de Venezuela (1995). Reglamento de los institutos y colegios universitarios. Gaceta Oficial $\mathrm{N}^{\circ}$ 4.992, extraordinario. Decreto $\mathrm{N}^{\circ} 865$. Caracas

Soto, A. (2000). Educación en tecnología. Un reto y una exigencia social. Bogotá: Cooperativa Editorial Magisterio

Toffler, A. (1993). El shock del futuro. Barcelona, España: Plaza y Janes Editores

UNESCO (1998). Documento de política para el cambio de la educación superior. Recuperado de https://unesdoc.unesco.org/ark:/48223/p fooooog8992_spa 